\title{
Does the Surface Matter? Hydrogen-Bonded Chain Formation of an Oxalic Amide Derivative in a Two- and Three-Dimensional Environment
}

\author{
Florian Klappenberger, ${ }^{*[a]}$ Marta E. Cañas-Ventura, ${ }^{[\mathrm{c}]}$ Sylvain Clair, ${ }^{[\mathrm{c}]}$ Stéphane Pons, ${ }^{[\mathrm{c}]}$ \\ Uta Schlickum, ${ }^{[\mathrm{cl}}$ Zhi-Rong Qu, ${ }^{[\mathrm{b}]}$ Thomas Strunskus, $^{[\mathrm{d}]}$ Alessio Comisso, ${ }^{[\mathrm{e}]}$ Christof Wöll, ${ }^{[\mathrm{d}]}$ \\ Harald Brune, ${ }^{[\mathrm{c}]}$ Klaus Kern, ${ }^{[\mathrm{c}]}$ Alessandro De Vita, ${ }^{[\mathrm{e}]}$ Mario Ruben, ${ }^{[\mathrm{bb}]}$ and Johannes V. Barth*[a]
}

\begin{abstract}
We report on a multi-technique investigation of the supramolec ular organisation of $\mathrm{N}, \mathrm{N}-$ diphenyl oxalic amide under differently dimensioned environments, namely three-dimensional (3D) in the bulk crystal, and in two dimensions on the Ag(111) surface as well as on the reconstructed Au(111) surface. With the help of Xray structure analysis and scanning tunneling microscopy (STM) we find that the molecules organize in hydrogen-bonded chains with the bonding motif qualitatively changed by the surface confinement. In two dimensions, the chains exhibit enantiomorphic order even though they consist of a racemic mixture of chiral en-
\end{abstract}

tities. By a combination of the STM data with near-edge X-ray absorption fine-structure spectroscopy, we show that the conformation of the molecule adapts such that the local registry of the functional group with the substrate is optimized while avoiding steric hindrance of the phenyl groups. In the low coverage case, the length of the chains is limited by the $A u(111)$ reconstruction lines restricting the molecules into fcc stacked areas. A kinetic Monte Carlo simulated annealing is used to explain the selective assembly in the fcc stacked regions.

\section{Introduction}

The spontaneous formation of nanostructures by molecular self-assembly is a promising way for the formation of future materials and devices. ${ }^{[1]}$ One of the major non-covalent interactions of the assembly process is hydrogen bonding, which provides selectivity and directionality. ${ }^{[2]}$ For two-dimensional (2D) patterning, molecular building blocks that were already successfully employed in three-dimensional (3D) supramolecular chemistry are often used. ${ }^{[3-7]}$ Systems based on planar molecules have been reported to build up the same bonding motifs in 2D as well as in 3D environments both for homomolecular ${ }^{[8-10]}$ and for heteromolecular cases. ${ }^{[5,6,11]}$ The resulting surface-confined architectures are influenced by the substrate on which the assembly takes place. ${ }^{[12]}$ The wide range of possible substrate-induced effects spans from moderate quantitative variations to qualitatively new ordering behavior. For example, hydrogen-bond lengths generally tend to be larger within networks on metal substrates than in crystal structures of the corresponding molecules. ${ }^{[3,8,10,13-15]}$ The restriction to a substrate not only modifies existing chiral properties, but novel and interesting chiral effects that are not possible for bulk materials also arise. ${ }^{[7,14,16-18]}$ Moreover, the interaction with the metal substrate can dictate a new conformation that differs remarkably from the geometry exhibited in the crystal phase and changes the functionality, as often experienced for porphyrins $^{[19]}$ and adaptive peptide or amino acid species. $^{[7,20,21]}$

Herein we report a multi-technique study of an oxalic amide derivative featuring the functional amide group that potentially forms hydrogen-bond motifs pivotal for the tertiary struc-

tures of a large number of biomolecules, in particular peptides. ${ }^{[22]}$ To obtain a deeper understanding of the transcription to $2 \mathrm{D}$, we systematically follow the hydrogen-bonded chain formation under different environments, namely in the crystal phase, on the $\mathrm{Ag}(111)$ surface, and on the reconstructed Au(111) surface. We also analyze the influence of the "bulky" phenyl substituents close to the functional group. The change from three to two dimensions induces novel chiral properties and a new type of hydrogen-bond motif connected with a

\footnotetext{
[a] Dr. F. Klappenberger, Prof. J. V. Barth

Physik Department E20

TU München, Munich (Germany)

Fax: $(+49) 8928912338$

E-mail:fklapp@ph.tum.de jvb@ph.tum.de

[b] Dr. Z.-R. Qu, Dr. M. Ruben Institute of Nanotechnology

Forschungszentrum Karlsruhe, Karlsruhe (Germany)

Fax: $(+49)$ 724782-643

E-mail:Mario.Ruben@int.fzk.de

[c] Dr. M. E. Cañas-Ventura, Dr. S. Clair, Dr. S. Pons, Dr. U. Schlickum Prof. H. Brune, Prof. K. Kern Institut de Physique des Nanostructures Ecole Polytechnique Fédérale de Lausanne CH-1015 Lausanne (Switzerland)

[d] Dr. T. Strunskus, Prof. C. Wöll Lehrstuhl für Physikalische Chemie I Ruhr-Universität Bochum, Bochum (Germany)

[e] Dr. A. Comisso, Dr. A. De Vita King's College London Strand, London WC2R 2 LS (UK)
} 
conformational adaptation. For submonolayer coverage on the Au substrate, chain ordering is governed by the surface reconstruction.

\section{Results and Discussion}

s-trans

Scheme 1. The $N, N$-diphenyl oxalic amide 1 in the planar trans configuration.

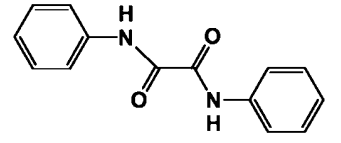

\subsection{Crystal Phase}

The synthesis of the $\mathrm{N}, \mathrm{N}$-diphenyl oxalic amide (Scheme 1) was first reported by Meyer et al. ${ }^{[23]}$ We resynthesized it, grew crystals from methanol/water solution, and found the same structural parameters as published earlier. ${ }^{[24]}$ The $X$ ray crystal structure demonstrates that the 3D phase consists of chains of molecules aligned along the direction a of the unit cell (Figure $1 \mathrm{a}$ ) and that each molecule is approximately aligned within the $\mathbf{b}-\mathbf{c}$ plane. Here the vectors $\mathbf{a}, \mathbf{b}$, and $\mathbf{c}$ define a right-handed orthogonal coordi-
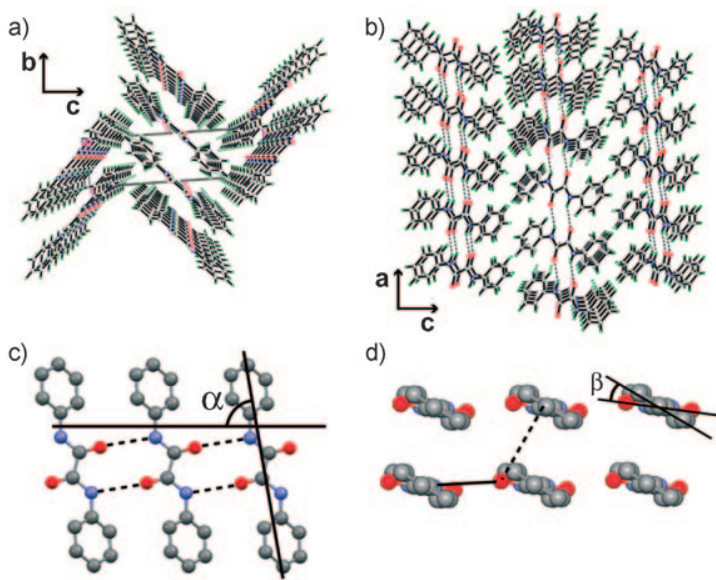

d)

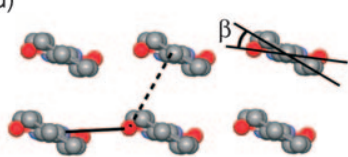

Figure 1. Crystal structure of diphenyl oxalic amide. a) View along direction $\mathbf{a}$ of the unit cell. b) View along direction $\mathbf{b}$. The molecules line up in chains via two hydrogen bonds (----) per molecule. Note that this view is achieved by rotating Figure 1 a by $90^{\circ}$ form top to bottom around the horizontal axis; thus molecules appear shorter due to the perspective projection. The left (right) end of the central chain is pointing out of (into) the paper plane. c) A view in the direction perpendicular to the plane of the functional group reveals the double $\mathrm{H}$-bonding pattern and the angle $\alpha$ of $80^{\circ}$. d) A view along the molecular axis highlights a rotation of the phenyl rings out of the plane of the functional group $\left(\beta=23^{\circ}\right)$.

nate system. Along the $\mathbf{c}$ direction the chains are ordered in a zigzag structure. A view along direction b (Figure $1 \mathrm{~b}$ ) highlights that the chains are held together by hydrogen bonding employing a complementary double bond motif of the $\mathrm{NH} \cdots \mathrm{O}$ type. Both hydrogen bonds are characterized by a bond length of $3.13 \AA$ for the $\mathrm{N} \cdots \mathrm{O}$ distance and $2.13 \AA$ for the $\mathrm{H} \cdots \mathrm{O}$ distance (Figure $1 \mathrm{c}$ ). We define the long molecular axis by connecting the two para-carbon atoms of the phenyl rings. The angle $\alpha$ between the long molecular axis and the direction of the chain amounts to $80^{\circ}$. A side view of the chains (Figure $1 \mathrm{~d}$ ) reveals that the tilt angle $\beta$ between the plane of the functional group of a molecule and the planes of the phenyl rings of the same molecule amounts to $23^{\circ}$. Because of the intermolecular hydrogen bonds (Figure $1 \mathrm{~d},-$ ) the functional oxalic amide groups are almost aligned within the same plane. The residual $7^{\circ}$ out-of-plane tilt evidences a chain stacking interaction to the next higher and lower chains that is governed by an attractive force (Figure $1 \mathrm{~d},----$ ) between the $\pi$ system of a phenyl in one chain and the electron attracting oxygen in an adjacent plane.

\subsection{Chain Formation on Surfaces}

Scanning tunneling microscopy (STM) data of a submonolayer coverage of 1 on $\mathrm{Ag}(111)$ demonstrate (Figure 2) that chain formation is expressed under the restriction to two dimensions as
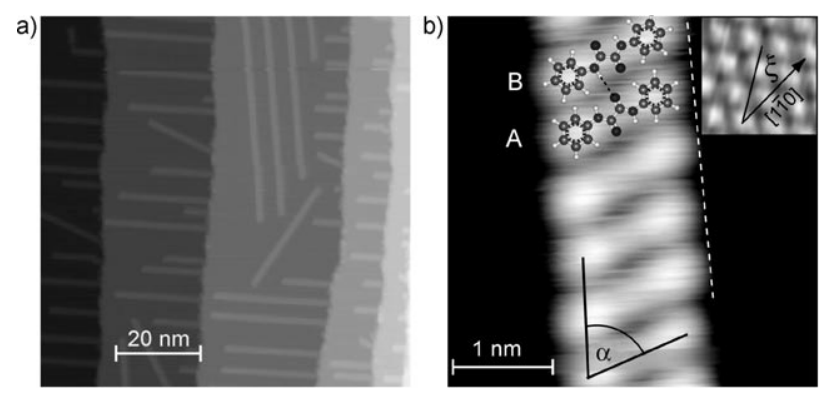

Figure 2. STM images after deposition of $0.3 \mathrm{ML}$ of 1 on $\mathrm{Ag}(111)$ held at $330 \mathrm{~K}$. a) Chains longer than $50 \mathrm{~nm}$ form along six directions (five of them are seen in this image). [Imaging parameters: $V_{\mathrm{b}}=-0.65 \mathrm{~V}, I=0.5 \mathrm{nA}$.] b) A high-resolution image $\left(V_{\mathrm{b}}=-0.8 \mathrm{~V}, I=0.33 \mathrm{nA}\right)$ indicates a smaller angle $\alpha$ $\left(70^{\circ}\right)$ than in the crystal phase. All chains show the same periodicity. Their directions are known with respect to the high-symmetry directions of the underlying $\mathrm{Ag}$ lattice (inset) and given by the angles $\xi$ (see Table 1). A proposed model based on molecules with alternating $2 D$ chirality, $A$ and $B$, is superimposed.

well. Molecules appear as protrusions and herein are displayed with bright contrast. Straight lines exhibiting different lengths of up to $50 \mathrm{~nm}$ are visible. The chains often start at the lower part of a step edge. Five different directions can be found in Figure $2 \mathrm{a}$. An analysis of a series of images evidences that six chain directions are present overall. In high-resolution images, the molecules appear as two bright lobes connected by a thinner waist (Figure $2 \mathrm{~b}$ ). The chain is $2 \mathrm{D}$ chiral as the image mirrored along an axis parallel to the chain direction cannot be obtained by a rotation around the surface normal. We define a chain to be of chirality $R$ if it is the right-hand side of the molecule that is in front when going along the chain. For all six directions of the molecular wires, their angles $\xi$ with respect to the [110] direction defined in the inset of Figure 2 were measured and listed in Table 1 together with their chirality label. Starting form the top of Figure $2 b$, every second molecule is imaged a bit shorter than the previous one (as can be seen from their not touching the dashed line connecting the ends of the longer molecules), indicating two distinct species (labeled $A$ and $B$ ). 


\begin{tabular}{|c|c|c|c|c|c|c|}
\hline Direction & $3 \mathrm{~L}$ & $2 \mathrm{R}$ & $1 \mathrm{~L}$ & $1 \mathrm{R}$ & $2 \mathrm{~L}$ & $3 R$ \\
\hline$\xi$ model & $10.1^{\circ}$ & $49.1^{\circ}$ & $70.1^{\circ}$ & $109.1^{\circ}$ & $130.1^{\circ}$ & $169.1^{\circ}$ \\
\hline$\xi \mathrm{Ag}(111)$ & $10^{\circ}$ & $48^{\circ}$ & $70^{\circ}$ & $110^{\circ}$ & $130^{\circ}$ & $169^{\circ}$ \\
\hline$\xi A u(111)$ & $10^{\circ}$ & $49^{\circ}$ & $69^{\circ}$ & $109^{\circ}$ & $132^{\circ}$ & $171^{\circ}$ \\
\hline \multirow[t]{3}{*}{ Periodicity } & \multicolumn{2}{|c|}{ Model } & \multicolumn{2}{|c|}{$\mathrm{Ag}(111)$} & \multicolumn{2}{|c|}{$\mathrm{Au}(111)$} \\
\hline & \multicolumn{2}{|c|}{$13.24 \AA$} & \multicolumn{2}{|c|}{$13.2 \AA$} & \multicolumn{2}{|c|}{$13.0 \AA$} \\
\hline & \multicolumn{6}{|c|}{$\mathrm{Au}(111)$} \\
\hline \multicolumn{2}{|c|}{$\mathrm{Nr}$ of chains } & & & 1 & \multicolumn{2}{|c|}{12} \\
\hline \multicolumn{2}{|c|}{ Average length } & & & 3.5 & & 5 \\
\hline
\end{tabular}

The two moieties can differ in conformation and/or in the local substrate registry. From the absence of a long-range Moiré pattern we conclude that the chains are commensurate with the substrate having a two-molecule repeat unit. The angle $\alpha$ amounts to $70^{\circ}$ and is thus $10^{\circ}$ smaller than in the crystal phase. The change of $\alpha$ renders the bonding motif of the crystal phase unfavorable as, in its homochiral chains, the $\mathrm{N}-\mathrm{H}$ groups of adjacent molecules would point towards each other and no $\mathrm{H}$ bonds would be formed. Instead, we propose that chains consist of a racemic mixture of molecules employing $2 D$ chiralities $A$ and $B$ on the $A g(111)$ surface (see Figure $2 \mathrm{~b}$ ). The oxalic amide becomes chiral when confined to the surface, even if it presents no chirality in the gas or crystal phase. Further evidence for the racemic mixture is provided by the experimental results on $\mathrm{Au}(111)$ and from calculations.

All chains have the same periodicity of $13.2 \AA$. For simplicity and taking into account that $\pi$-conjugated organic molecules often adopt a planar adsorption geometry, ${ }^{[3,5,8,17]}$ we firstly hypothesize a planar conformation and superimpose models of molecules of types A and B onto the STM image, the size of which is calibrated with the atomic resolution of the plain $\mathrm{Ag}(111)$ surface (inset). This preliminary model indicates that only one hydrogen bond of a $\mathrm{NH}$... $\mathrm{O}$ type is present, which features a $\mathrm{N} \cdots \mathrm{O}(\mathrm{H} \cdots \mathrm{O})$ distance of $3.7 \AA(2.7 \AA)$. Furthermore, a separation of only $1.5 \AA$ between the nearest $\mathrm{H}$ atoms of neighboring phenyl rings follows from this simple model. This distance is in the repulsive range of intermolecular forces, which means that our assumption of a planar conformation cannot describe the real adsorption geometry reasonably well. Thus a non-planar conformation is likely.

The pristine $A u(111)$ surface exhibits a reconstruction of the first layer consisting of the stacking of 23 surface atoms on 22 bulk hollow sites along the $\langle 1 \overline{1} 0\rangle$ directions. ${ }^{[25]}$ The reconstruction leads to parallel corrugation lines ${ }^{[26]}$ along $\langle 11 \overline{2}\rangle$ that divide the surface into regions with fcc-type and hcp-type stacking. The locally uniaxial contraction is converted into an overall isotropic contraction by a periodic bending of the corrugation lines by $120^{\circ}$ (chevron pattern). The reconstruction manifests itself in STM images as doubled zigzag lines that appear brighter than the rest of the surface (dark gray lines in Figure 3).

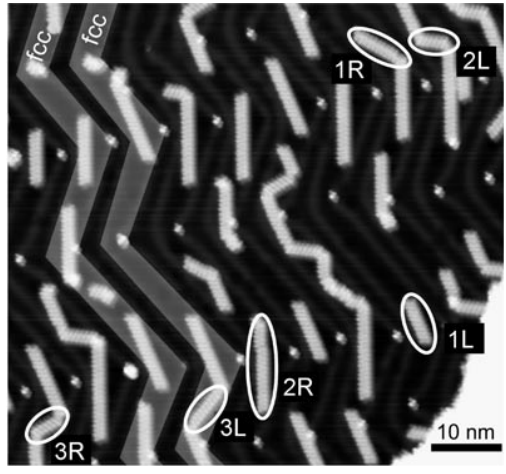

Figure 3. STM topograph (recorded at $9 \mathrm{~K}$ ) of $0.2 \mathrm{ML}$ of 1 deposited on a $\mathrm{Au}(111)$ surface held at $330 \mathrm{~K}$ shows one-dimensional chains (bright gray) with an average length of 12 molecules. The chains appear exclusively in the fcc stacking regions (highlighted) featuring six directions, three of each chirality $\mathrm{R}$ and $\mathrm{L}$. $\left(I=0.26 \mathrm{nA}, V_{\mathrm{B}}=-0.3 \mathrm{~V}\right.$.)

The deposition of approximately $0.2 \mathrm{ML}$ (Figure 3 ) of the oxalic amide derivative onto a $\mathrm{Au}(111)$ surface held at $330 \mathrm{~K}$ and subsequent cooling down to imaging conditions results in the formation of short chain segments $(10 \mathrm{~nm}$, molecules appear bright) with a small separation (approximately $5 \mathrm{~nm}$ ) and not in extended chains with a correspondingly larger separation. Thus, the chain formation is qualitatively changed in comparison to the behavior found on the unreconstructed $\mathrm{Ag}$ surface. At low coverage, chains appear exclusively in the fcc stacking regions as highlighted in the left part of Figure 3. There are chains in six different directions, and three of each of the two chiralities, indicating that only the length of the chains is limited by the reconstruction, whereas the orientational order with respect to the local registry is retained. However, the combination of molecules occupying only fcc regions with the directions of the chains differing from the directions of the reconstruction lines results in a correlation of chain direction and average length. Table 1 shows that the variants $2 R$ and $1 \mathrm{~L}$ build the longest chains with an average length of 14 molecules, whereas the other four directions have a shorter average length. From Figure 3, it is evident that the directions $2 \mathrm{R}$ and $1 \mathrm{~L}$ profit from the fact that they decorate the elbow sites and extend into the fcc regions to both sides. These numbers correspond to one domain of the Au reconstruction; at a macroscopically different place, similar images rotated by $120^{\circ}$ could be obtained where, for example, the directions $2 \mathrm{~L}$ and $3 R$ would exhibit the longest average chain length.

The phenomenon of molecules preferring fcc regions on the $\mathrm{Au}(111)$ surface has been reported for several cases, but so far no satisfactory explanation has been found. ${ }^{[27]}$ However, modeling the observed structures described above suggests a possible interpretation. Notably, this is not based on assuming that a net difference of adsorption energy may provide the driving force for the selective observation of fcc-supported chains. Rather, we show that the steric constraint of shorter chaining imposed by the narrower hcp stacked regions of the substrates is a sufficient condition to explain the observed behavior. This picture is revealed by a series of combined first- 
principles electronic structure calculations and a Monte Carlo annealing of chain nucleation and growth.

Although the absolute value of the density functional theory (DFT) adsorption energy is sensitive to the choice of the exchange and correlation functional used, energy differences are usually calculated with much higher precision. We therefore expect any significant difference in the molecular adsorption energy on the two differently stacked substrates to be revealed by these calculations. However, at $0.03 \mathrm{eV}$ per molecule, the calculated adsorption energy difference falls within the range of error of the theory, and should thus be regarded as negligible. This excludes an effect coming from geometric stacking, which is reasonable, since fcc and hcp domains differ only after the third atomic layer. However, the surface-state local density of states (LDOS) has been reported to differ significantly between the two stacking areas. ${ }^{[28,29]}$ This difference is concentrated close to energies of the surface state onset. The LDOS integrated from -0.51 to $-0.45 \mathrm{eV}$ with respect to the Fermi energy, $E_{\mathrm{F}}$, varies by $40 \%$. Integrating up to $-0.32 \mathrm{eV}$ reduces this variation to $20 \%$. It goes further down as one approaches $E_{\mathrm{F}}$, so that the total charge density in the surface state only shows a tiny variation. ${ }^{[28]}$ Significant differences in binding energy are only expected if the molecules are especially sensitive to the LDOS between -0.5 and $-0.4 \mathrm{eV}$ with respect to $E_{\mathrm{F}}$, whereas a much broader energy range for the interaction between the molecules and the surface is indicated in our calculations. Thus, the differences expected from the different electronic structures are small. However, it is beyond the scope herein, and possibly pushing beyond the state-of-the-art of DFT, to perform calculations on a slab sufficiently large to host the reconstruction, and sufficiently precise to reveal these differences. We therefore assume that the electronic structure differences discussed above have no significant influence on the binding energy. Instead, we provide the following picture rationalizing the observed preference of the chains for fcc stacking areas.

The striking imbalance in the population of the two domains may be explained by steric reasons connected to the finite maximum size of the chains observed in the STM images, related to the chain termination energy cost. To investigate this possibility, we carried out a Monte Carlo simulation of the annealing process performed in the experiments before acquiring the STM images, taking the different sizes of the domains into account directly. The experimental results (Figure 3 ) reveal that no molecular chain continues across the boundary between the fcc- and hcp-stacked zones. On a few occasions, when more molecules are present locally than the maximum number that can be placed while forming straight lines in fcc regions, chains form kinks to remain contained inside fcc zones rather than continuing straight across the boundary. Without kink formation, the characteristic zigzag pattern of the chevron reconstruction, the lateral thickness of the molecular chains and the lateral size of the reconstruction zones determine the maximum length of any single straight chain segment within each stacked region (cf. Figure 3 ). Since the fcc-stacked regions are laterally larger than the hcp ones, molecular chains of 20 and
10 molecules are allowed to form on the fcc and hcp regions, respectively.

We implemented a Monte Carlo annealing simulation based on the following simple rules: 1) molecular chains can nucleate at the elbow sites of both fcc and hcp regions, with an equal number of possible chains for the two reconstructed regions on the surface, 2) we fix a maximum number $N$ of molecules for each chain, which is different for chains growing on the two different reconstruction domains, 3) in a single Monte Carlo step, a terminal molecule can move from any existing chain to any other allowed site of the surface, either joining another chain or leading to the nucleation of a new one, 4) the energy $\Delta E$ spent for detaching a molecule from a $N$-molecule long chain (with $N>1$ ) is equal to the energy gained by attaching the molecule to a pre-existing chain, regardless of the type of stacking underneath. Rule 1 is suggested by the fact that adsorption energy differences are negligible on the two substrates (as suggested by our DFT results). Rule 2 corresponds to the experimental fact that the chains are always observed to stop at the boundary between the two stacking regions (the possibility that chains occasionally form "elbows" is neglected for simplicity). Rules 3-4 simply express thermodynamic equilibrium: the Monte Carlo simulation obtained by these rules correspond to monomer exchanges between molecular chains (at zero overall energy cost), and nucleation events on previously empty nucleation sites (at the energy cost $\Delta E$ ). The only difference between the two populations of chains growing on fcc and hcp stacking regions is that their chain length is capped at significantly different maximum numbers $N_{\text {fcc }}$ and $N_{\text {hcp }}$ of molecules. From the geometry of the herringbone reconstruction and after inspection of the STM images we set $N_{\text {fcc }}=20, N_{\text {hcp }}=10$. The only remaining parameter of the calculation is set as $\Delta E=0.085 \mathrm{eV}$, half the intermolecular binding energy obtained by the DFT calculations, and used in the standard Metropolis algorithm rejection rule while annealing.

The results for a typical run are displayed in Figure 4, showing two sample configurations of a system containing $35 \mathrm{fcc}$ and 35 hcp nucleation sites hosting about 500 molecules, which corresponds to an experimental coverage of $0.1 \mathrm{ML}$. The annealing process was done in steps of $10 \mathrm{~K}$, and the two snapshots are taken at about $300 \mathrm{~K}$ (Figure $4 \mathrm{a}$ ) and $7 \mathrm{~K}$ (Figure $4 \mathrm{~b}$ ). While at a temperature comparable with the truncation energy of the chains, both populations are visible. The different maximum length constraints for the two types of chains is such that chain fluctuations eventually lead to the disappearance of hcp chains even within the limited amount of Monte Carlo steps $\left(\approx 10^{6}\right)$ accessible by these calculations.

This result suggests that the kinetics of the assembly is such that the system is capable to reach its structural minimum energy state during the experimental annealing. This produces the geometry which maximizes the total intermolecular number of bonds by assembling all the molecules in the minimum overall number of chains. Thus, these are of the longer fcc type. Crucial factors, rendering possible the extinction of the hcp type, are: 1) the relatively short chain lengths determined by the reconstruction domain size and shape con- 

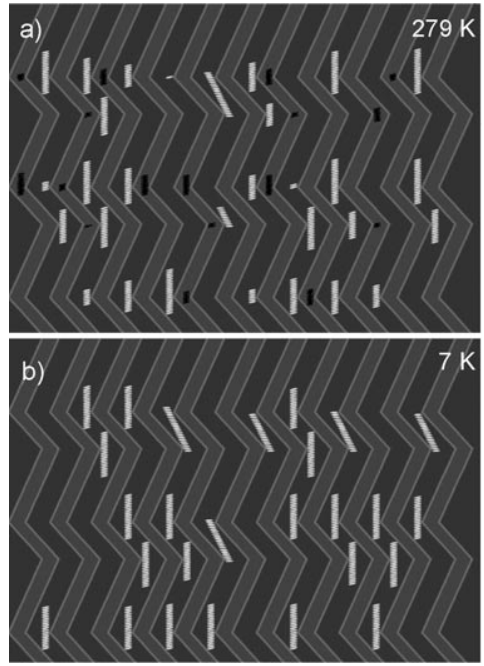

Figure 4. Simulated annealing of the chain growth for an adlayer coverage of $0.2 \mathrm{ML}$. Two sample configurations of the chain distribution in thermodynamic equilibrium are presented. a) At room temperature some chains are present in the hcp domains (black on light grey), but the majority still occupies the fcc domains (white on dark grey). B) At the imaging temperature $(7 \mathrm{~K})$ only the fcc domains are populated.

straints allowing fluctuation-trigged extinction of hcp chains (approximate probability $2^{-10}$ ) and 2 ) the fact that the maximum chain length allowed on the two domains are distinctively different, making the fcc chains much more resistant against fluctuation-triggered extinction (probability $2^{-20}$ ). Hereby, the extinction probabilities were calculated assuming that a terminal molecule can either jump to a different chain or rest at its place with equal probability $(0.5)$ as a result of the same energy for both possibilities. When a chain of length $n$ is extinguished for $n$ times in a row, the terminal molecule jumps to a different chain, which happens with a probability of $2^{-n}$.

The fluctuation-trigger extinction process can be interpreted as a 2D interface analog of the Ostwald ripening process taking place in precipitation from supersaturated solution. ${ }^{[30]}$ There, small particles are dissolved and large particles grow larger due to the enhanced solubility of the small particles. In our case, the metal surface plays the role of the transport medium (solution) and the increased solubility of the small particles is represented by the higher extinction probability of the small chains.

On both surfaces we have found chains in six directions. Their chiral properties are discussed next. Our analysis of the local registry of the supramolecular structures with the substrate is summarized in Figure 5. It is known ${ }^{[25,26]}$ that the double reconstruction lines (brighter red) are oriented along a $\langle 11 \overline{2}\rangle$ direction (white arrow in Figure $5 \mathrm{a}$ ). The chains to both sides demonstrate that the latter direction is a symmetry axis. When connecting the ends of the molecules of one chain, a zigzag ordering becomes apparent (black line). Taking into account that no Moiré pattern is visible, we conclude a commensurate superstructure with two molecules in the unit cell. The measured periodicity is $13.0 \pm 0.5 \AA$. We reported earlier ${ }^{[21]}$ that both the racemic mixture in the unit cell and the single hydro-

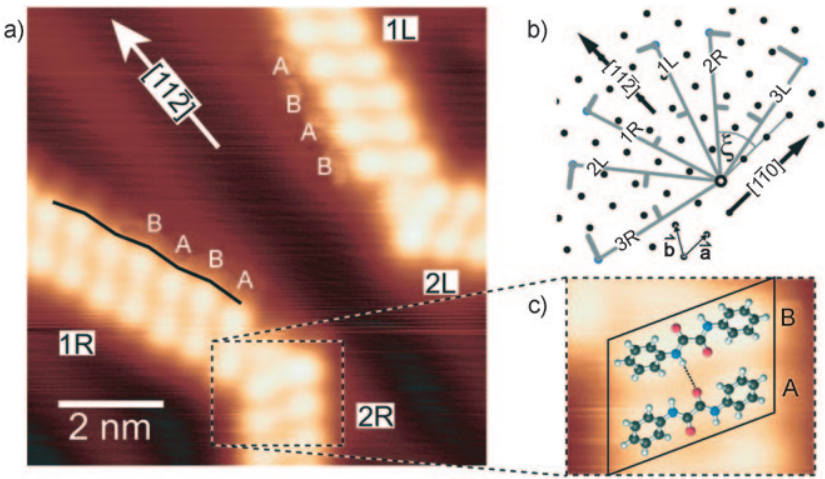

Figure 5. a) A close-up image of molecular chains in directions ( $1 R$ and $2 R$ ) with their chiral counterparts ( $1 \mathrm{~L}$ and $2 \mathrm{~L}$ ). The [112] direction (indicated by the arrow) acts as the mirror axis. A slight zigzagging is visible when connecting the ends of the molecules. $\left(I_{T}=0.2 \mathrm{nA}, V_{B}=-0.3 \mathrm{~V}\right.$.) b) A sketch of the relation between the local surface atom arrangement and the different chain directions. c) Tentative model shown on a zoomed part (-----) of Figure $5 \mathrm{a}$. The rhombic unit cell $(-)$ contains a racemic mixture of the two chiral entities $A$ and $B$.

gen-bond motif are confirmed by DFT calculations. Within our experimental resolution, the assembly on the $A u(111)$ surface is the same as on the $\mathrm{Ag}(111)$ surface. Thus, we propose an identical model to explain the chain formation for both substrates. Defining $\vec{a}$ and $\overrightarrow{\mathbf{b}}$ as the primitive translation vectors in directions $[1 \overline{1} 0]$ and $[10 \overline{1}]$, respectively, and with the nearest neighbor length $(2.889 \AA)$, six commensurate directions $\left[\left(\begin{array}{l}4 \\ 1\end{array}\right),\left(\begin{array}{l}1 \\ 4\end{array}\right),\left(\begin{array}{c}-1 \\ 5\end{array}\right),\left(\begin{array}{c}-4 \\ 5\end{array}\right),\left(\begin{array}{c}-5 \\ 4\end{array}\right),\left(\begin{array}{c}-5 \\ 1\end{array}\right)\right]$ with a length of $13.24 \AA$ are found (Figure $5 \mathrm{~b}$ ). The corresponding angles with respect to the [110] direction are listed in Table 1 . Both the angles and the periodicities found in the experiment agree well with the model. For the $\mathrm{Au}(111)$ substrate, the reconstruction induces compression along the [110] direction. Thus, the atomic distances as well as the angles should be changed in comparison with the silver, but most of the short lines on the gold traverse the elbow sites where the compression changes its direction and is less pronounced. Thus, on average, the compression is negligible for the supramolecular assemblies.

We studied the case of an almost complete monolayer of the oxalic amide derivative on the reconstructed $\mathrm{Au}(111)$ surface. A large-scale image displays four terraces (Figure 6a) where each terrace is strongly dominated by chains in one direction. The tendency to align in one direction is strongest on the narrow terraces; on wider terraces domains with different preferred directions are found. Directions $2 \mathrm{R}$ and $1 \mathrm{~L}$ appear more often than the other four, indicating that during the growth those directions in which longer and more chains are formed, trigger the preferred direction of the resulting domain. The elbow-shaped double lines of the surface reconstruction are visible through the adlayer (black lines on terrace 2). The chains are crossing the reconstruction lines, which is even more evident in an enlargement (Figure $6 \mathrm{~b}$ ) where the pairing of chains also becomes apparent. For high coverage, the space-filling principle together with the boundary conditions imposed by the terraces are energetically more demanding 

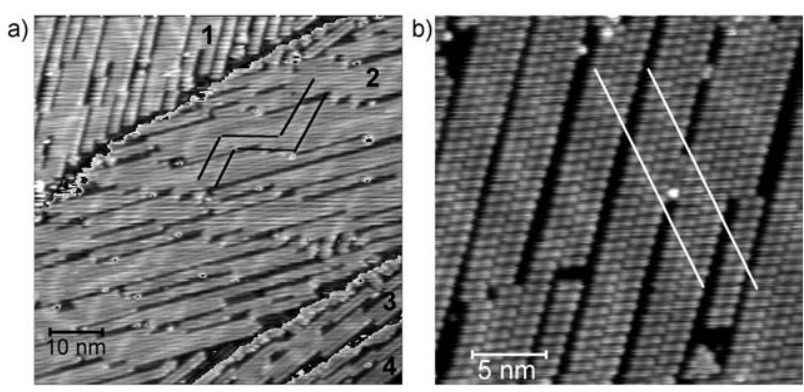

Figure 6. STM topographs of an almost saturated monolayer of the oxalic amide on $\mathrm{Au}(111)$. a) Each of the four terraces (1-4) is covered with chains in one direction crossing the gold reconstruction, with examples indicated by black double lines. A repeated grayscale is used to give the same contrast for each terrace. $\left(I_{T}=0.5 \mathrm{nA}, V_{\mathrm{B}}=-0.83 \mathrm{~V}\right.$.) b) A zoom on one terrace highlights that the bonding motif is identical with that for chains forming at lower coverage and that only one chirality of the chains is present on one terrace. $\left(I_{\mathrm{T}}=0.5 \mathrm{nA}, V_{\mathrm{B}}=1 \mathrm{~V}\right.$.)

than complying with the fcc-hcp difference. More importantly, an analysis of the chain properties reveals the same angles $\xi$ and $\alpha$ as well as the same chain periodicity as in the submonolayer case, thus no coverage-dependent reorganization of bond motifs or molecular conformation is indicated.

\subsection{Conformational Adaptation}

With the help of near-edge X-ray absorption fine-structure (NEXAFS) spectroscopy we studied the conformation of the oxalic amide in two different environments, namely in a multilayer and a monolayer sample. The multilayer was prepared by evaporating approximately 10 adlayers of molecules onto a clean $\mathrm{Au}(111)$ single crystal held at $180 \mathrm{~K}$ during evaporation. The saturated monolayer sample was achieved by $10 \mathrm{~min}$ evaporation onto a newly cleaned $\mathrm{Au}(111)$ sample at $330 \mathrm{~K}$, as adsorption is terminated after the first layer completion under these conditions.

A semi-empirical extended Hückel calculation of the molecular orbitals of $\mathbf{1}$ in a conformation near to that obtained from the crystal structure shows that the lowest unoccupied molecular orbital (LUMO) is a $\pi^{*}$ orbital concentrated on the oxalic amide groups (Figure $7 \mathrm{a}$ ), whereas the LUMO +1 has its electron density on the phenyl rings (Figure $7 \mathrm{~b}$ ). Thus, by analyzing the corresponding resonances into these final states, we obtain two independent measures for the different molecular

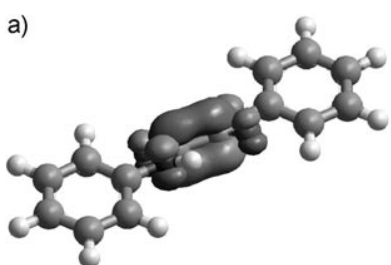

LUMO

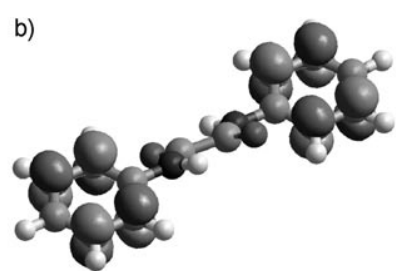

$\mathrm{LUMO}+1$
Figure 7. Extended Hückel calculation of the a) LUMO and b) LUMO +1 for a conformation close to the one obtained by X-ray structure analysis, showing orbitals with their molecular weights concentrated on the central oxalic amide and the terminal phenyl groups, respectively. moieties. Hereby we exclude the possibility of a nonplanar amide backbone based on the fact that the crystal structure as well as all our calculated geometry-optimized structures always have a planar backbone. For both cases, the direction of the orbitals is perpendicular to the plane of the corresponding group. For a given NEXAFS peak, its intensity dependence on the incidence angle $\theta$, between the surface normal and the $E$-vector of the X-ray beam, characterizes the geometric orientation of the corresponding molecular moiety. We define the orientation angle $\gamma$ as the angle between the surface normal and the direction of the final state orbital connected to the peak.

The $\mathrm{C} 1 \mathrm{~s}$ region for the multilayer sample (Figure $8 \mathrm{a}$ ) features three sharp peaks in the $\pi^{*}$ range $(285-290 \mathrm{eV})$ and broad structures in the $\sigma^{*}$ range. The first peak is attributed to the
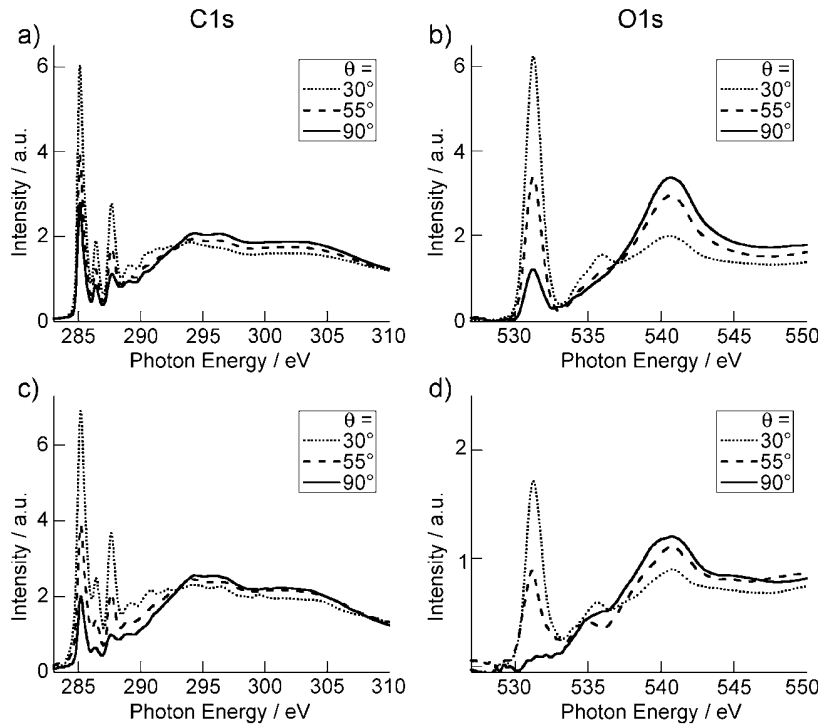

Figure 8. NEXAFS study of the molecular orientation for a multilayer ( $a$ and b) and a saturated monolayer ( $c$ and d) of the oxalic amide on $\mathrm{Au}(111)$. The multilayer data are consistent with the crystal conformation obtained by Xray structure analysis. In the monolayer case a flat-lying functional group is indicated by the $01 \mathrm{~s}$ spectra. The $\mathrm{C} 1 \mathrm{~s}$ region shows that the phenyl rings are rotated approximately $30^{\circ}$ with respect to the functional group, thus $\beta$ is larger under the influence of the surface than in the crystal phase.

lowest $\pi^{*}$ resonance of the phenyl rings based on its energy close to that of benzene on $\mathrm{Au}(111)^{[31]}$ and due to its strong relative intensity. The angle dependence of this phenyl resonance yields $\gamma=40^{\circ}$. The orientation of the oxalic amide moiety was obtained from the $01 \mathrm{~s}$ spectra shown in Figure $8 \mathrm{~b}$. Here the lowest-lying resonance $(531.2 \mathrm{eV})$ is attributed to a transition into the $\pi^{*}$ orbital that originates from the LUMO. The angle dependence indicates $\gamma=30^{\circ}$. The peak at $541 \mathrm{eV}$ is attributed to the $\sigma^{*}$ resonance of the carbonyl group and is oriented along the $\mathrm{C}-\mathrm{O}$ axis.

A comparison to the previous case shows that the features of the monolayer sample (Figures $8 \mathrm{c}$ and $\mathrm{d}$ ) appear at very similar energies and with only a slight increase in their widths, qualifying the molecule-substrate interaction to be physisorption or at most weak chemisorption. The most obvious change 
is the vanishing intensity for normal incidence $\left(\theta=90^{\circ}\right)$ in the O1s region, indicating an oxalic amide group oriented coplanar to the surface (Figure $8 \mathrm{~d}$ ). The analysis of the $\mathrm{C} 1 \mathrm{~s}$ peak related to the phenyl rings yields an orientation angle $\gamma=30^{\circ}$. Taking into account that the functional group is parallel to the surface, a tilt angle $\beta=30^{\circ}$ can easily be concluded for the molecular conformation within chains in the monolayer on the closepacked gold surface.

The determination of the conformation in the multilayer is not as straightforward as that for the monolayer case. There are two extreme ways to interpret the orientation of the oxalic amide part $\left(\gamma=30^{\circ}\right)$ together with a set of intermediate possibilities. Starting from an alignment parallel to the substrate surface, the central group can be rotated by $30^{\circ}$ either around the long molecular axis (case A) or around an axis perpendicular to the long molecular axis and parallel to the carbonyl axis (case B). The first case would rotate the carbonyl axis out of the surface plane and thus decrease the dichroism of the $\sigma^{*}$ resonance at $541 \mathrm{eV}$. The second case would rotate the phenyl rings out of the surface plane, but leave the carbonyl axis oriented within the surface plane and thus leave the $\sigma^{*}$ dichroism pronounced as it is. Thus the combination of the two 01s resonances indicates an orientation of the functional group near to case B.

In a sufficiently thick multilayer the influence of the metal substrate is expected to vanish and the molecules should adopt bulk properties. We test whether the NEXAFS data can be interpreted in terms of a tilt angle $\beta$ equal to the crystal phase. To do so, we assume that the molecule exhibits a completely planar conformation and that it is oriented within the $x-y$ plane, aligned along the $x$-axis with the long molecular axis. Now we rotate the phenyl rings by $23^{\circ}$ around the $x$-axis to obtain the crystal phase conformation. Then we rotate the entire molecule around the $y$-axis by $30^{\circ}$ to align the inner group according to the NEXAFS data (case B). For such an arrangement, a NEXAFS measurement of $\gamma_{\text {phenyl }}$ would yield $37^{\circ}$. Thus the multilayer spectra agree with the assumption of a conformation similar to the one found in the crystal phase.

Thus, confinement to two dimensions affects the tilt of the phenyl rings in a counterintuitive way. The physisorptive interaction of extended $\pi$ systems of organic molecules with noble metal surfaces results from dispersive forces, ${ }^{[32]}$ but is typically strong enough to orient the $\pi$ systems flat on the surface. Here, the increase in $\beta$ indicates that the energy gained by avoiding the steric hindrance of the phenyl rings overcomes the energy gain due to the interaction of the phenyl $\pi$ system with the metal substrate. Thus, on the surface, a planar conformation, as suggested in the preliminary model in Figure $2 \mathrm{~b}$, does not prevail. This observation is qualitatively confirmed by the DFT calculations. However, the phenyl-substrate interaction is overestimated by the chosen Perdew-Wang 92 LDA functional, leading to smaller tilt angles of $10^{\circ}$, as reported elsewhere. ${ }^{[21]}$

From the fact that the substrate-induced conformation of the organic monolayer does not influence the multilayer conformation, we infer that the molecular flexibility allows compensation of the different boundary conditions imposed by crystal growth from solutions and by thin-film growth on a metal substrate within a few monolayers.

The capability of adapting to different local premises while keeping up the chain formation is similarly expressed in the formation of irregular "worm-like" chain arrangements as seen in Figure 3 at the right of the center. Such chain properties based on molecules exhibiting conformational flexibility allow for formation of amorphous systems on a different basis compared to those systems realized with cytosine on $\mathrm{Au}(111)$, where several elementary structural binding motifs of rigid molecules are incorporated in a supramolecular random network. ${ }^{[33]}$ Rather, the present observations are related to the coexistence of several tiling motifs obtained with the rubrene molecule on $\mathrm{Au}(111){ }^{[34]}$ where conformational freedom was suggested to afford organizational variety, but direct evidence for conformational changes was missing. A conformational adaptation similar to the one found here also played an important role herein during the assembly of linear molecules into a chiral Kagomé lattice. ${ }^{[18]} \mathrm{A}$ qualitative change of the bonding type resulting in unconventional motifs was equally encountered in metal-directed assembly at surfaces. ${ }^{[35]}$

\section{Conclusions}

We demonstrated how steric constraints influence the self-assembly of an exemplary flexible oxalic amide species. We employed complementary experimental and theoretical methods to analyze the formation of hydrogen-bonded one-dimensional molecular wires in different three- and two-dimensional environments. While the expected twofold $\mathrm{H}$-bond chaining motif appears in the organic crystallites, the need to comply with the local registry of the close-packed noble surfaces triggered chiral chains of racemic mixtures of molecules featuring a qualitatively new bond motif with only one hydrogen bond between molecules. Thereby we identified a conformational adaptation that is necessary to circumvent steric hindrance of the phenyl rings in the surface-supported chains. In contrast to the situation on the homogenous $\mathrm{Ag}(111)$ surface, the $\mathrm{Au}(111)$ reconstruction controls the chain nucleation sites and their length for small enough coverage. The preferential assembly in fcc stacking regions is associated with fluctuation-triggered extinction of the shorter hcp chains. Altogether, our results contribute to an improved understanding and control of supramolecular organization of adaptive species on surfaces.

\section{Experimental Section}

The scanning tunneling microscope (STM) experiments were performed in an UHV system (base pressure in the low $10^{-11} \mathrm{mbar}$ range) equipped with standard sample preparation facilities and a home-built low-temperature STM. ${ }^{[36]}$ Images were taken in the constant current mode at $10 \mathrm{~K}$ using a $\mathrm{W}$ tip. Indicated voltages correspond to sample bias, thus, tunneling takes place from occupied tip states into unoccupied sample states for positive bias. The surfaces were cleaned by repeated ion gun sputtering at room temperature with an Argon flux of approximately $7 \mu \mathrm{Acm}^{-2}$ and annealing to $740 \mathrm{~K}$ and $890 \mathrm{~K}$ for the $\mathrm{Ag}$ and Au crystals, respectively. The molecules were deposited from a quartz crucible in a Knud- 
sen-type cell held at $410 \mathrm{~K}$ onto the metal surface at temperatures as indicated. One monolayer (ML) is defined as an adlayer fully covering the surface. NEXAFS measurements were performed at the BESSY II HE-SGM beamline. All spectra were taken at a sample temperature of $160 \mathrm{~K}$ with a slit width of $200 \mu \mathrm{m}$ corresponding to an energy resolution of $0.4 \mathrm{eV}$ for the $\mathrm{C} 1 \mathrm{~s}$ edge and $0.7 \mathrm{eV}$ for the $\mathrm{O} 1 \mathrm{~s}$ edge. The raw data were treated to concentrate on the adlayer-related information; the details on data processing are described elsewhere. ${ }^{[15]}$

We carried out the theoretical investigation of $N, N$-diphenyl oxalic amide adsorbed on the $\mathrm{Au}(111)$ surface employing first-principles electronic structure techniques and molecular dynamics simulations using the Car-Parrinello method. We used the PerdewWang 92 local density approximation for representing exchange and correlation interactions of the valence orbitals. ${ }^{[37]}$ The planewave expansion cut-off was set to $28 \mathrm{Ry}$ and the Brillouin zone sampling in all cases was limited to the gamma point only. A $0.25 \mathrm{eV}$ Fermi level smearing width was used to treat the metallic slab system, as described elsewhere. ${ }^{[38]}$ All the calculations used periodic boundary conditions. A vacuum region of $10 \AA$ was used to minimize the interaction between each gold slab and its periodically repeated images.

\section{Acknowledgements}

This work was partially supported by the European Science foundation EUROCORES-SONS-FunSmarts network. The INFN-DEMOCRITOS and UTS-CENMAT Centers, Trieste (Italy) as well as the CNR are gratefully acknowledged. Traveling costs for synchroton measurements provided by the BMBF through Grant No. 05ES3XBA 15 are gratefully acknowledged.

Keywords: conformations $\cdot$ hydrogen bonds · scanning probe microscopy $\cdot$ self-assembly $\cdot$ supramolecular chemistry

[1] D. Philp, J. F. Stoddart, Angew. Chem. 1996, 108, 1242-1286; Angew. Chem. Int. Ed. Engl. 1996, 35, 1155-1196; J. M. Lehn, Proc. Natl. Acad. Sci. USA 2002, 99, 4763-4768.

[2] G. R. Desiraju, Acc. Chem. Res. 2002, 35, 565-573; T. Steiner, Angew. Chem. 2002, 114, 50-80; Angew. Chem. Int. Ed. 2002, 41, 48-76; L. J. Prins, D. N. Reinhoudt, P. Timmerman, Angew. Chem. 2001, 113, 24462492; Angew. Chem. Int. Ed. 2001, 40, 2382-2426.

[3] J. V. Barth, J. Weckesser, C. Z. Cai, P. Gunter, L. Burgi, O. Jeandupeux, K. Kern, Angew. Chem. 2000, 112, 1285; Angew. Chem. Int. Ed. 2000, 39 1230.

[4] R. Otero, M. Schöck, L. M. Molina, E. Laegsgaard, I. Stensgaard, B. Hammer, F. Besenbacher, Angew. Chem. 2005, 117, 2310-2315; Angew. Chem. Int. Ed. 2005, 44, 2270-2275; C. Z. Cai, B. Müller, J. Weckesser, J. V. Barth, Y. Tao, M. M. Bösch, A. Kündig, C. Bosshard, I. Biaggio, P. Günter, Adv. Mater. 1999, 11, 750-754; S. Stepanow, N. Lin, F. Vidal, A. Landa, M. Ruben, J. V. Barth, K. Kern, Nano Lett. 2005, 5, 901-904; M. Stöhr, M. Wahl, C. H. Galka, T. Riehm, T. A. Jung, L. H. Gade, Angew. Chem. 2005, 117, 7560-7564; Angew. Chem. Int. Ed. 2005, 44, 73947398.

[5] J. A. Theobald, N. S. Oxtoby, M. A. Phillips, N. R. Champness, P. H. Beton, Nature 2003, 424, 1029-1031.

[6] M. E. Cañas-Ventura, W. Xiao, D. Wasserfallen, K. Müllen, H. Brune, J. V. Barth, R. Fasel, Angew. Chem. 2007, 119, 1846-1850; Angew. Chem. Int. Ed. 2007, 46, 1814-1818.

[7] M. Lingenfelder, G. Tomba, G. Costantini, L. C. Ciacchi, A. De Vita, K. Kern, Angew. Chem. 2007, 119, 4576-4579; Angew. Chem. Int. Ed. 2007, $46,4492-4495$.
[8] A. Dmitriev, N. Lin, J. Weckesser, J. V. Barth, K. Kern, J. Phys. Chem. B 2002, 106, 6907-6912.

[9] S. Griessl, M. Lackinger, M. Edelwirth, M. Hietschold, W. M. Heckl, Single Mol. 2002, 3, 25-31; M. Ruben, D. Payer, A. Landa, A. Comisso, C. Gattinoni, N. Lin, J.-P. Collin, J.-P. Sauvage, A. De Vita, K. Kern, J. Am. Chem. Soc. 2006, 128, 15644; M. Lackinger, S. Griessl, T. Markert, F. Jamitzky, W. M. Heckl, J. Phys. Chem. B 2004, 108, 13652-13655.

[10] S. Clair, S. Pons, A. P. Seitsonen, H. Brune, K. Kern, J. V. Barth, J. Phys. Chem. B 2004, 108, 14585-14590.

[11] S. Xu, M. Dong, E. Rauls, R. Otero, T. R. Linderoth, F. Besenbacher, Nano Lett. 2006, 6, 1434.

[12] S. M. Barlow, R. Raval, Surf. Sci. Rep. 2003, 50, 201-341; F. Rosei, M. Schunack, Y. Naitoh, P. Jiang, A. Gourdon, E. Laegsgaard, I. Stensgaard, C. Joachim, F. Besenbacher, Prog. Surf. Sci. 2003, 71, 95-146; J. V. Barth, G. Costantini, K. Kern, Nature 2005, 437, 671-679; J. V. Barth, Annu. Rev. Phys. Chem. 2007, 58, 375-407.

[13] M. Vladimirova, G. Trimarchi, A. Baldereschi, J. Weckesser, K. Kern, J. V. Barth, A. De Vita, Acta Mater. 2004, 52, 1589-1595.

[14] J. V. Barth, J. Weckesser, G. Trimarchi, M. Vladimirova, A. D. Vita, C. Cai, H. Brune, P. Günther, K. Kern, J. Am. Chem. Soc. 2002, 124, 7991-8000.

[15] M. E. Cañas-Ventura, F. Klappenberger, S. Clair, S. Pons, K. Kern, H Brune, T. Strunskus, C. Wöll, R. Fasel, J. V. Barth, J. Chem. Phys. 2006 $125,184710$.

[16] M. Ortega Lorenzo, C. J. Baddeley, C. Muryn, R. Raval, Nature 2000, 404, 376-379; K. H. Ernst, Top. Curr. Chem. 2006, 265, 209-252; R. Fasel, M. Parschau, K. H. Ernst, Angew. Chem. 2003, 115, 5336-5339; Angew. Chem. Int. Ed. 2003, 42, 5178-5181; M. C. Blüm, E. Cavar, M. Pivetta, F. Patthey, W. D. Schneider, Angew. Chem. 2005, 117, 5468-5471; Angew. Chem. Int. Ed. 2005, 44, 5334-5337; R. Fasel, M. Parschau, K. H. Ernst, Nature 2006, 439, 449-452; S. Weigelt, C. Busse, L. Petersen, E. Rauls, B. Hammer, K. V. Gothelf, F. Besenbacher, T. R. Linderoth, Nat. Mater. 2006, 5, 112-117.

[17] F. Vidal, E. Delvigne, S. Stepanow, N. Lin, J. V. Barth, K. Kern, J. Am. Chem. Soc. 2005, 127, 10101-10106.

[18] U. Schlickum, R. Decker, F. Klappenberger, G. Zoppellaro, S. Klyatskaya, W. Auwärter, S. Neppl, K. Kern, H. Brune, M. Ruben, J. V. Barth, J. Am. Chem. Soc. 2008, 130, 11778-11782.

[19] V. Arima, E. Fabiano, R. I. R. Blyth, F. Delia Sala, F. Matino, J. Thompson, R. Cingolani, R. Rinaldi, J. Am. Chem. Soc. 2004, 126, 16951-16958; T. Yokoyama, T. Kamikado, S. Yokoyama, S. Mashiko, J. Chem. Phys. 2004, 121, 11993-11997; W. Auwärter, F. Klappenberger, A. Weber-Bargioni, A. Schiffrin, T. Strunskus, C. Wöll, Y. Pennec, A. Riemann, J. V. Barth, J. Am. Chem. Soc. 2007, 129, 11 279-11 285; D. Bonifazi, A. Kiebele, M. Stohr, F. Y. Cheng, T. Jung, F. Diederich, H. Spillmann, Adv. Funct. Mater. 2007 17, 1051-1062; H. Wende, M. Bernien, J. Luo, C. Sorg, N. Ponpandian, J. Kurde, J. Miguel, M. Piantek, X. Xu, P. Eckhold, W. Kuch, K. Baberschke, P. M. Panchmatia, B. Sanyal, P. M. Oppeneer, O. Eriksson, Nat. Mater. 2007, 6, 516-520; A. Weber-Bargioni, W. Auwarter, F. Klappenberger, J. Reichert, S. Lefrancois, T. Strunskus, C. Woll, A. Schiffrin, Y. Pennec, J. V. Barth, ChemPhysChem 2008, 9, 89-94.

[20] A. Kühnle, T. R. Linderoth, B. Hammer, F. Besenbacher, Nature 2002, 415 891-893; A. Schiffrin, A. Riemann, W. Auwärter, Y. Pennec, A. Weber-Bargioni, D. Cvetko, A. Cossaro, A. Morgante, J. V. Barth, Proc. Natl. Acad. Sci. USA 2007, 104, 5279-5284; M. Weinhold, S. Soubatch, R. Temirov, M. Rohlfing, B. Jastorff, F. S. Tautz, C. Doose, J. Phys. Chem. B 2006, 110, 23756-23769; G. Tomba, M. Lingenfelder, G. Costantini, K. Kern, F. Klappenberger, J. V. Barth, L. C. Ciacchi, A. De Vita, J. Phys. Chem. A 2007 111, 12740-12748; L. M. Ghiringhelli, L. Delle Site, J. Am. Chem. Soc 2008, 130, 2634-2638

[21] F. Klappenberger, M. E. Cañas-Ventura, S. Clair, S. Pons, U. Schlickum, Z. R. Qu, H. Brune, K. Kern, T. Strunskus, C. Wöll, A. Comisso, A. De Vita, M. Ruben, J. V. Barth, ChemPhysChem 2007, 8, 1782-1786.

[22] K. A. Dill, Biochemistry 1990, 29, 7133-7155; D. L. Nelson, M. M. Cox Lehninger Principles of Biochemistry, 5th ed., Freeman, New York, 2007

[23] R. Meyer, A. Seeliger, Ber. Dtsch. Chem. Ges. 1896, 29, 2640-2645.

[24] Y. H. Wen, X. M. Li, L. Wang, Acta Crystallogr. Sect. E 2006, 62, 02185; S. Liesecke, H. Görls, private communication.

[25] J. V. Barth, H. Brune, G. Ertl, R. J. Behm, Phys. Rev. B 1990, 42, $9307-$ 9318.

[26] C. Wöll, S. Chiang, R. J. Wilson, P. H. Lippel, Phys. Rev. Lett. 1989, 39, 7988. 
[27] M. Böhringer, K. Morgenstern, W. D. Schneider, M. Wühn, C. Wöll, R. Berndt, Surf. Sci. 2000, 444, 199-210; W. D. Xiao, P. Ruffieux, K. Ait-Mansour, O. Groning, K. Palotas, W. A. Hofer, P. Groning, R. Fasel, J. Phys. Chem. B 2006, 110, 21394-21398; Z. H. Cheng, L. Gao, Z.T. Deng, N. Jiang, Q. Liu, D. X. Shi, S. X. Du, H. M. Guo, H. J. Gao, J. Phys. Chem. C 2007, 111, 9240-9244; I. Fernandez-Torrente, S. Monturet, K. J. Franke, J. Fraxedas, N. Lorente, J. I. Pascual, Phys. Rev. Lett. 2007, 99, 176103.

[28] L. Bürgi, H. Brune, K. Kern, Phys. Rev. Lett. 2002, 89, 176801.

[29] W. Chen, V. Madhavan, T. Jamneala, M. F. Crommie, Phys. Rev. Lett. 1998 80, 1469-1472.

[30] W. Ostwald, Z. Phys. Chem. Stoch. Verwandtschaftsl. 1900, 34, 495-503.

[31] K. Weiss, S. Gebert, M. Wühn, H. Wadepohl, C. Wöll, J. Vac. Sci. Technol. A 1998, 16, 1017-1022.

[32] A. Bilic, J. R. Reimers, N. S. Hush, R. C. Hoft, M. J. Ford, J. Chem. Theory Comput. 2006, 2, 1093-1105.

[33] R. Otero, M. Lukas, R. E. A. Kelly, W. Xu, E. Laegsgaard, I. Stensgaard, L. N. Kantorovich, F. Besenbacher, Science 2008, 319, 312-315.
[34] M. Pivetta, M. C. Blum, F. Patthey, W. D. Schneider, Angew. Chem. 2008 120, 1092-1095; Angew. Chem. Int. Ed. 2008, 47, 1076-1079.

[35] U. Schlickum, R. Decker, F. Klappenberger, G. Zoppellaro, S. Klyatskaya, M. Ruben, I. Silanes, A. Arnau, K. Kern, H. Brune, J. V. Barth, Nano Lett. 2007, 7, 3813-3817; S. Stepanow, N. Lin, D. Payer, U. Schlickum, F. Klappenberger, G. Zoppellaro, M. Ruben, H. Brune, J. V. Barth, K. Kern Angew. Chem. 2007, 119, 724-727; Angew. Chem. Int. Ed. 2007, 46, 710 713; S. Stepanow, N. Lin, J. V. Barth, J. Phys. Condens. Matter 2008, 20, 184002.

[36] S. Clair, Ph.D. thesis thesis, Ecole Polytechnique Federale de Lausanne 2004

[37] J. P. Perdew, Y. Wang, Phys. Rev. B 1992, 45, 13244-13249.

[38] M. Stengel, A. De Vita, Phys. Rev. B 2000, 62, 15283-15286.

Received: September 7, 2008

Published online on November 7, 2008 